\title{
Control of parallel computing in a cyber-physical production environment
}

\author{
Anatoly V. Shukalov ${ }^{1}$, Igor O. Zharinov ${ }^{1}$ and Oleg O. Zharinov ${ }^{2}$ \\ ${ }^{1}$ Faculty of Information Security and Computer Technologies, ITMO University, 49, Kronverksky Av., Saint \\ Petersburg, 197101, Russia \\ ${ }^{2}$ Department of Problem-Oriented Computing Complexes, Saint Petersburg State University of Aerospace \\ Instrumentation, 67, Bolshaya Morskaia str., Saint Petersburg, 190000, Russia
}

\begin{abstract}
The problem of the functional stability of the Industry 4.0 production facility is considered. The structure of cyber-physical system is given, the components of which are controlled through a special purpose network. The communication features of the software control of equipment are determined. The regulation of production processes is provided by built-in automation that supports tools for online modeling of technological processes. Equipment management is performed in an optimization model adequate for parallel computing systems. Communication control mechanisms of cyber-objects are borrowed from the theory of automatic systems and from the theory of networks. Joint calculations allow controlling industrial equipment through the network and adjusting the coefficients of regulators responsible for tuning cyber-physical systems. Improving the manageability of cyber-physical production is based on the modification of command and data flows, adaptively changing for technological tasks. Multipurpose control is implemented through a network that transfers targeted regulatory actions to controlled objects. The presence of computing, control and network components ensure the functional stability of the processes of physical dynamics of production, monitored by software.
\end{abstract}

\section{Keywords}

Parallel computing, cyber-physical production, Industry 4.0, Internet of Things

\section{Introduction}

The Industry 4.0 digital plant technical equipment segment problem point requires to create a new generation control system, which uses some approaches and cyber-physical equipment to unite technological processes with programmable production environment $[1,2]$. A completely new point being implemented with cyber-physical systems (CPS) to integrate logical and physical environment is to put in order the item manufacturing processes in a plurality of logically independent control tides [3, 4].

A cyber-physical production (CPP) is designed as a net of autonomous robots, which receive and transmit independently the timetable to characterize physical processes, which happen in parallel in different CPSs [5]. The necessary CPS working organs functioning is controlled through a net with a production system configurator, which use communications of Wi-Fi, 4G, EDGE and other. The distributed dynamic structures interaction is based on the production technological parameters measurements and evaluations, which are controlled with two control systems [6, 7]:

- $\quad$ an automatic closed loop, which controls electronically CPS technological processes integrated into a production machine and checking exploitation parameters correctness with programmed algorithms (end machine model with control micro-system);

III International Workshop on Modeling, Information Processing and Computing (MIP: Computing-2021), May 28, 2021, Krasnoyarsk, Russia

EMAIL aviation78@mail.ru (Anatoly V. Shukalov); mpbva@mail.ru (Igor O. Zharinov); zharinov73@inbox.ru (Oleg Zharinov)

ORCID: 0000-0003-0283-5207 (Anatoly V. Shukalov); 0000-0003-2508-5939 (Igor O. Zharinov); 0000-0003-1219-8205 (Oleg Zharinov)

(c) (i) (C) 2021 Copyright for this paper by its authors.

Use permitted under Creative Commons License Attribution 4.0 International (CC BY 4.0).

CEUR Workshop Proceedings (CEUR-WS.org) 
- an automatic closed loop, which controls with a software CPS information process and distributed among plant functional elements components (which emulates and checks the CPP normal operation with a control macro-system).

A CPP multi-aspect control concept is done with program and electronic systems, which control the CPS output signals values in each information tide which has an IP (Internet Protocol)-agent [8, 9]. The

CPS initiation and control are provided with different route technologies engaging short and long cycles [10]:

- the analogue control short cycle is done in by-operation CPS automatics control mode oriented to complete production processes in real time;

- the digital control long cycle is done through a net and related to the CPS virtual regulation engaging technological operations correction mechanisms, which are done in production environment model time.

To make a decision how to control a non-stationary production processes beyond the CPS physical limits is an element how to develop the integrated calculation system with a normal appearance for an industrial object. The CPS distributed remote control pretends today to be an innovation, which makes closer the hardly compatible technologies implementation for the CPP project infrastructure [11, 12$]$.

A problem how to change the Industry 3.0 base technologies is researched today with many articles. The authors stress the digital control system positive role with company resources, which converge innovations into industry. Also, a majority of given results is of mainly fragmentary character, which does not cover the CPP full functionality [13]. The more authoritative approach to create a digital plant in the Industry 4.0 planning horizon is to synthesize a three-level concept of a CPP, which combines the laws of uninterrupted and discrete CPS structural dynamics control with the information processes software control analytical tools $[14,15]$.

\section{The CPP software configuration}

The CPP construction methodology is associated with industrially made cyber-systems implementations and their technology to the smart factory infrastructure without human personal presence necessity in the object. The CPP dynamic system is a combination of connected to the company nets physical objects, which are capable to form data congregations and translate them to the virtual control service. A part of technological data being processed is done with digital signal processors calculation resources integrated into first technical CPS devices. The second data processing parameters (signatures) are transmitted through the net to the CPP intellectual accompanying centers containing control applications. The scheme of interaction of the CPS with the components of the CPP environment is shown in Figure 1:.

The calculation analytics plural integration complex model and production process require to unite CPSs into Internet of Things (IoT) sub-nets and equipment groups, which control autonomously their technological operations. Each CPS device role which is a modem net access points carrier is configured with integrated software and corrected with cybernetics amendments from the control center to reflect smart factory properties and conditions in the viewed moments of time.

General and applicable CPSs functions, which is the base of CPP functionality sequences are related to the Industry 4.0 three components technological equipment specifics, which contains:

- the CPS calculation component to complete analytical work with data congregations and item manufacturing given scenarios, which are described with the item digital twin formats and processes;

- the CPS communication component to complete the electric and informative company nets integration, which are heterogeneous traffic physical transmission channels with standardized protocols;

- the CPS control component to complete the plant equipment adaptive processes regulation automatizing with controllable technological parameters in uninterrupted aggregates dynamic interaction production cycle. 


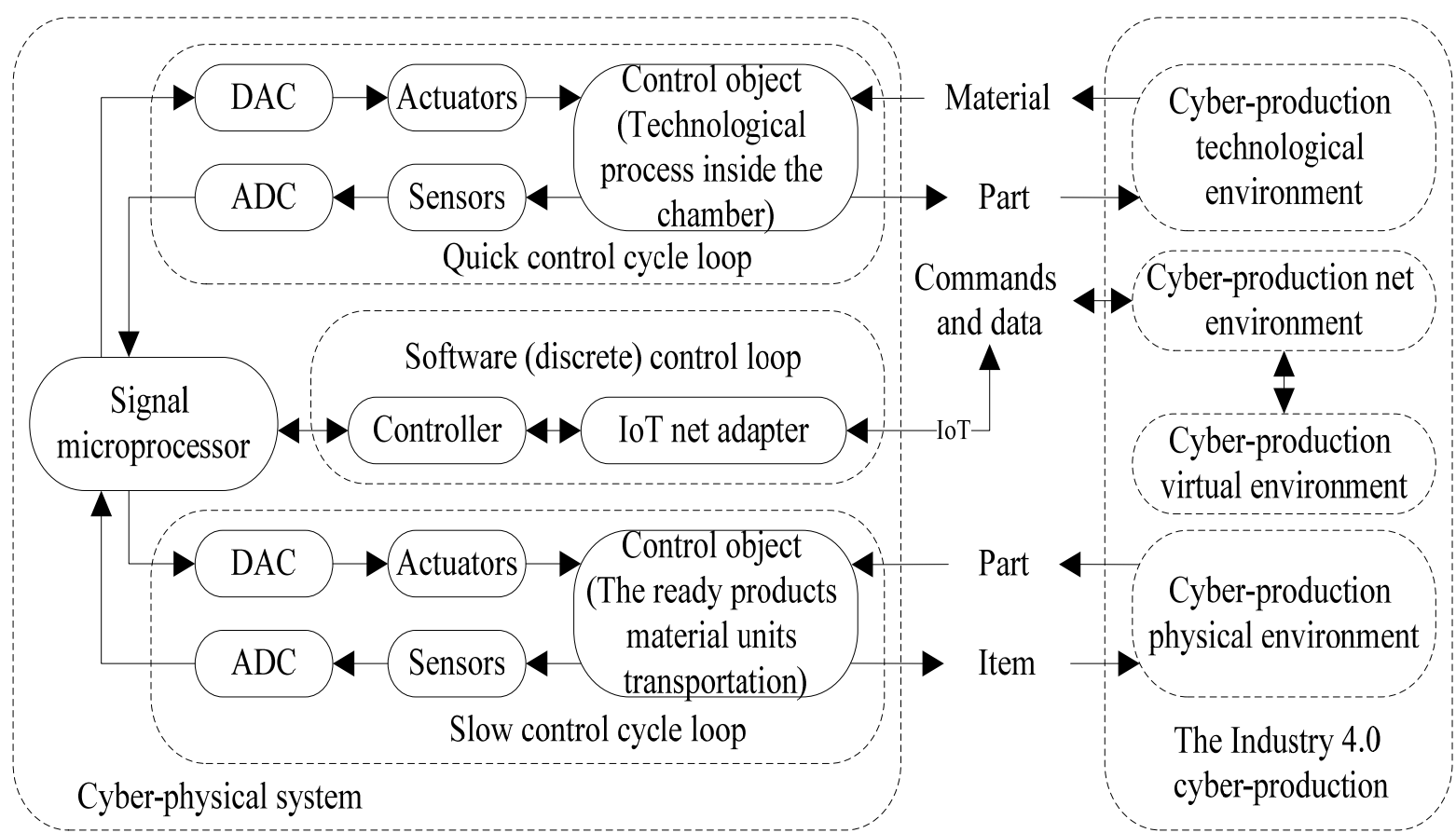

Figure 1: The scheme of interaction of the CPS with the components of the CPP environment (ADC analog to digital converter, DAC - digital to analog converter)

The CPS exploitation conditions, which influence the sub-systems measurement accuracy and being regulated parameters control are constantly being monitored with a computerized CPP environment using telecommunication contact means. The plant environment CPS being prognosticated behavior is provided with software applications and operational systems distributed in a CPP:

- the platform dependent CPS software functioning under operational systems of Linux, Android,

IOS, Windows NT and other control (it is used in the end CPS physical devices level);

- the platform independent CPS software supports the codes of JavaScript, html-page, xml-text and other high-level languages, which are used in the company cloud environment server resources.

\section{The CPS exchange timing discrete control}

The CPP equipment conflict overcoming (the resources access collision) is done with production tasks solution tools, which use a relay system of protective automatics. The CPS exploitation potentially possible modes reaching, which are attractive to construct a smart factory based on informative and technological integration of aggregates physical and virtual components. The cross-platform solution base is a wireless CPP communication, which translate controlling signals and data among CPS end devices and the cloud environment in both directions.

The package traffic exchange is a factor of dynamic production system vitality to provide CPS automatic resilience. The CPS net components inter-connection is done with mechanization means (machine solution) and radiofrequency information exchange means (software solution). The CPS initial working modes setting-up is after the equipment initializing stage in the company digital infrastructure:

- the CPS machine initializing in the CPP net is based on the agent MAC (Media Access Control)-address identification defined with physical lines adapters integrated into each aggregate;

- the CPS program initializing in the CPP net activates the aggregate digital twin in the company cloud environment and gives IP addresses to the physical and virtual agents.

The CPS modes setting-up is provided with controlling variables and program components being downloaded from a cloud storage. The CPP full functionality is supported in two ways how to compile everything being executed with a signal processor and program codes controller: 
- $\quad$ static compiling when the API (Application Programming Interface)-library functions, which are integrated into file application file (which are used to program automatics systems integrated in a CPS and which regulates technological parameters);

- dynamic compiling when the library content is related to the company virtual environment and are called with CPS program means remotely (which are used for the main control system of multicomponent CPP).

The CPS technological modes adaptive online control is done with discrete and uninterrupted automatics sub-systems functioning under appearing disturbances:

- control analogue signals are formed in a high accurate automatics coil to service the item manufacturing processes and parameters regulation, which pass in CPS autonomously;

- digital control signals are worked with cloud applications, which translate variable through an IoT net for production processes remote regulation.

The signal formats transformation and production data signatures coding, which circulate on a net and significantly reduce CPS preparation coefficient for uninterrupted functioning as an industrial object. The CPS end device net traffic processing delay could appear in following situations:

- CPS maintenance refusal because the net messages exchange buffer is overflowed or there is a conflict of data information tides;

- error appearance in the radio channel data transmission because of the signal forms frequency distortions of the being monitored CPSs in an IoT net over different electromagnetic disturbances;

- CPS net connection session awaiting because of volume traffic transmission (for example, software distributives) between the control center and end agents and other.

The CPS device discrete control, which must be done under synchronizing with time reference for automatics digital elements, which calculate the desired regulation parameters values. For a CPP with technological lines longitude factor the reference time signals to tact the control process are made with a server, which gives second marks timing the CPS autonomous exchange. Being transmitted in the net variables are corresponded in a CPS with single CPP time signals to guarantee the control packages synchronizing and their restoration after losses related to the physical item manufacturing processes.

\section{Conclusion}

An ultra-modern strategic plan how to develop the Industry 4.0 means to create a CPP company, which corresponds the resilience and controllability principles and equipped with functional equipment of extended capabilities. To approximate the intellectual control system to the item manufacturing physical processes replaces the traditional production organization logic, which requires a fixed technological equipment structure in an industrial object. The technological task solution to control the Industry 4.0 is based on the dynamically changing quantity reconfigurable structures and a number of CPS specialties for the end-product manufacturing requirements.

The Industry 4.0 sense aspects are oriented for real science progress for automatized systems technologies with an unbreakable connection to the CPSs production assets control problem. The closed automatics systems create a plurality of control and regulation loops, which coordinate CPP technological processes completion. Keeping in production relationships participants mind dynamic complexity of control laws factors are made with a number of intellectual technologies connected with an IoT and sufficient for the one-time control of distributed CPSs.

The CPP possible structures form after control system active components informative interaction redistributing technological data tides among CPSs. To improve integrated calculation systems technologies means to change controller programming logic, which permits the item manufacturing production parameters correction through a net. The CPS control system cyber-identical state are shown with physical devices digital twins to describe the technological equipment different aspects.

The same tine functioning physical and virtual components interaction is made through API functions program interfaces to define the net centering control technologies. The indirect CPS control is provided with a content (commands and data), which circulates between the executive mechanisms and the CPP intellectual core. In the IoT context theoretical and practical CPP significance are after the solutions, which let balance the CPS calculation load and company communication nets with asynchronous traffic being transmitted through a radio channel without distortions or losses. 
The IoT net agents programming language means and algorithms codes of their control defines the production logistics new format generated dynamically for each being manufactured item unit. To combine technologies and CPS equipment in the viewable Industry 4.0 perspective must be a part of company development strategy, which includes organization system changing for CPP control. The cyber-technologies base elements ready for use today are penetrated already into the industry and its new potential development is already pre-destined.

\section{References}

[1] S. Thiede, Procedia CIRP 69 (2018) 644-649.

[2] E. Trunzer, A. Wullenweber, B. Vogel-Heuser, Journal of industrial information integration 19 100155 (2020).

[3] M. Kra, S. Horbrand, J. Schilp, Procedia CIRP 81 (2019) 1160-65.

[4] A. V. Gurjanov, D. A. Zakoldaev, A. V. Shukalov, I. O. Zharinov, Journal of Physics: Conference Series 1059(1) 012010 (2018).

[5] M. Schreiber, K. Vernickel, C. Richter, G. Reinhart, Procedia CIRP 79 (2019) 534-539.

[6] M. Diani, M. Colledani, Procedia CIRP 91 (2020) 741-746.

[7] H. S. Kang, J. Y. Lee, Procedia CIRP 72 (2018) 462-467.

[8] Y. Lu, T. Peng, X. Xu, Computers \& industrial engineering 129 (2019) 56-66.

[9] V. Brandstetter, J. C. Wehrstedt, IFAC-PapersOnLine 51(11) (2018) 809-814.

[10] A. V. Gurjanov, D. A. Zakoldaev, A. V. Shukalov, I. O. Zharinov, Journal of Physics: Conference Series 1015(5) 052035 (2018).

[11] N. Kshetri, Telecommunications policy 44(8) 102007 (2020).

[12] C. Faller, M. Hoftmann, Procedia CIRP 67 (2018) 156-161.

[13] N. Nikolakis, V. Maratos, S. Makris, Robotics and computer-integrated manufacturing 56 (2019) 233-243.

[14] E. Negri, L. Fumagalli, C. Cimino, M. Macchi, Procedia manufacturing 28 (2019) 201-206.

[15] A. V. Gurjanov, D. A. Zakoldaev, A. V. Shukalov, I. O. Zharinov, IOP Conference Series: Materials Science and Engineering 327(2) (2018) 022111. 